\title{
MAPPINGS BETWEEN FUNCTION SPACES $\left({ }^{1}\right)$
}

BY

\author{
ROBERT G. BARTLE AND LAWRENCE M. GRAVES
}

In the following we shall let $\mathfrak{U}=[u]$ and $\mathfrak{B}=[v]$ denote arbitrary Banach spaces, and $\mathfrak{T}=[t]$ denote a Hausdorff space. $\mathfrak{X}$ and $\mathfrak{V}$ are to denote the spaces of all continuous functions mapping $\mathfrak{T}$ into $\mathfrak{U}$ and into $\mathfrak{B}$ respectively. We shall let $\Omega$ denote the space of all linear continuous mappings of $\mathfrak{u}$ into $\mathfrak{B}$. A function $K$ on $\mathfrak{T}$ to $\Omega$ which is bounded on $\mathfrak{I}$ and continuous in the strong topology of $\Omega$ induces a linear continuous operator $\kappa$ on $\mathfrak{X}$ to $\mathfrak{Y}$ by the formula $\left({ }^{2}\right)$

$$
\kappa(x \mid t)=K(t) x(t) .
$$

The main result of this paper, contained in $\$ 3$, gives sufficient conditions that $\kappa$ map $\mathfrak{X}$ onto the whole space $\mathfrak{Y}$. Applications to nonlinear equations, integral equations, and to underdetermined systems of differential equations, including certain types of partial differential equations, are given in $\S \S 4,5$, and 7. In $\$ 6$ we consider the case when $\mathfrak{T}$ is a compact interval of the real axis, and let $\mathfrak{X}^{\prime}$ and $\mathfrak{Y}^{\prime}$ denote the subspaces of $\mathfrak{X}$ and $\mathfrak{Y}$ consisting of those functions which are of class $C^{\prime}$. Sufficient conditions are given that $\kappa$ map $\mathfrak{X}^{\prime}$ onto $\mathfrak{Y}^{\prime}$, and this result is applied to the case of nonlinear equations.

In the case when, for each $t, K(t)$ gives a one-to-one correspondence between the spaces $\mathfrak{U}$ and $\mathfrak{B}$, the results of this paper have been long known and are easily derived. The methods used here enable us to prove the existence of solutions with certain useful properties in cases when the solution is not unique.

1. Topological preliminaries. J. A. Dieudonné has discussed [3] a generalization of the notion of compactness of a topological space which will be of considerable use in $\S 3$. We therefore summarize a few definitions and results in the form that we shall need. In the following all coverings of topological spaces are supposed to consist of open sets.

Definitions. A covering $\mathfrak{B}$ is said to be a refinement of the covering $\mathfrak{A}$ if every set in $\mathfrak{B}$ is contained in some set in $\mathfrak{A}$. A covering is said to be neighborhood-finite if every point has a neighborhood which intersects only a finite

Presented in part to the Society by the senior author, April 29, 1950; received by the editors August 9, 1951.

(1) The part contributed by the junior author was completed while he was an Atomic Energy Commission Predoctoral Fellow.

(2) The following remarks on notation may be useful. If $A$ is an element of $\Omega$, the functional value of $A$ for a given $u$ will be denoted by $A(u)$ or by $A u$ as convenient. When $A=K(t)$, $u=x(t)$, it is convenient to write $K(t) x(t)$. For fixed $x, \kappa(x)$ is a function of $t$, whose value at $t, \kappa(x)(t)$, is more conveniently written $\kappa(x \mid t)$. 
number of sets in the covering. A Hausdorff topological space is said to be paracompact if every covering $\mathfrak{A}$ has a refinement covering $\mathfrak{B}$ which is neighborhood-finite.

Dieudonné showed that every paracompact space is a normal $\left(T_{4}\right)$ topological space, and that every closed subset of a paracompact space is itself paracompact in its relative topology, but this is not generally true for arbitrary subsets. On the other hand, A. H. Stone showed [7] that every metric space is paracompact. This theorem is very important for our purposes. It implies, in particular, that any subset of a metric space is paracompact. It is not known whether there are any spaces other than metrizable spaces in which arbitrary subsets are paracompact.

We shall need the following special case of Theorem 6 in Dieudonnés paper. (Cf. also Lefschetz [8, p. 26].)

LEMmA. Let $\mathfrak{T}$ be a normal space and $\left\{A_{\nu}\right\}$ a covering of $\mathfrak{T}$ which is neighborhood-finite. Then there exists a covering $\left\{B_{\nu}\right\}$ of $\mathfrak{T}$ with the same index class and for which $\bar{B}_{\nu} \subset A_{\nu}$ for all $\nu$.

Under the conditions of this lemma, it follows that for each $\nu$ there exists a real-valued continuous function $\psi_{\nu}$ which is non-negative everywhere and such that $\psi_{\nu}(t)=1$ for $t \in B_{\nu}$ and $\psi_{\nu}(t)=0$ for $t \in A_{\nu}$. By the assumption of neighborhood-finiteness we know that every point is contained in only a finite number of the sets $\left\{A_{\nu}\right\}$, hence $\psi_{\nu}(t)=0$ except for a finite number of indices. Moreover each point is contained in some $B_{\nu}$, so not all of the functions can vanish at any given point. Hence the sum $\sum_{\nu} \psi_{\nu}(t)$ is well-defined at each point and is neither zero nor infinite. Since, in the neighborhood of each point, only a finite number of terms are nonzero, this sum is a continuous function on all of $\mathfrak{T}$. Let $\phi_{\nu}(t)=\psi_{\nu}(t) / \sum_{\nu} \psi_{\nu}(t)$. It is clear that $\phi_{\nu}(t)=0$ for $t \notin A_{\nu}$, and $\sum_{\nu} \phi_{\nu}(t)=1$ for all $t \in \mathfrak{T}$. Such a collection of continuous functions $\left\{\phi_{\nu}\right\}$ is called a continuous partition of unity subordinate to the covering $\left\{A_{\nu}\right\}[2$, p. 66].

The following lemma follows immediately from the above discussion:

Lemma. Let $\mathfrak{T}$ be a paracompact space and $\left\{C_{\mu}\right\}$ a covering of $\mathfrak{T}$. Then there is a neighborhood-finite refinement covering $\left\{A_{\nu}\right\}$ and a continuous partition of unity $\left\{\phi_{\nu}\right\}$ subordinate to $\left\{A_{\nu}\right\}$.

We note that $\left\{\phi_{\nu}\right\}$ is also subordinate to $\left\{C_{\mu}\right\}$ in the sense that for every $\nu$ there exists some $C_{\mu}$ outside of which $\phi_{\nu}$ is identically zero.

2. Induced linear transformations. In the space $\mathfrak{X}$ of all continuous functions on $\mathfrak{T}$ to $\mathfrak{U}$, we define the "norm" of an element $x$ by the formula $\|x\|=\sup \{\|x(t)\| \mid t \in \mathfrak{T}\}$. The subspace of $\mathfrak{X}$ on which this norm is finite we denote by $\mathfrak{X}_{B}$; it is a Banach space. When $\mathfrak{I}$ is sequentially compact $\mathfrak{X}_{B}=\mathfrak{X}$. The corresponding notations $\mathfrak{Y},\|y\|, \mathfrak{Y}_{B}$ are used in connection with the space of continuous functions on $\mathfrak{T}$ to $\mathfrak{B}$. 
In the space $\Omega$ of linear continuous transformations $L$ of $\mathfrak{U}$ into $\mathfrak{B}$, the "uniform" topology is that determined by the norm in $\Omega$, while the "strong" topology is that corresponding to a system of neighborhoods $V\left(0 ; \epsilon, u_{1}, \cdots, u_{n}\right)$ of the origin. Here $V\left(0 ; \epsilon, u_{1}, \cdots, u_{n}\right)$ consists of all transformations $L$ satisfying $\left\|L u_{i}\right\|<\epsilon, \quad i=1, \cdots, n$, where $\epsilon>0$ and $u_{1}, \cdots, u_{n}$ are arbitrary elements of $\mathfrak{u}$. We denote by $\Omega_{0}$ the subset of $\Omega$ consisting of all the transformations mapping $\mathfrak{U}$ onto all of $\mathfrak{B}$.

In this section we investigate certain relations between functions $K$ mapping $\mathfrak{I}$ into $\Omega$, and linear continuous transformations $\kappa$ of $\mathfrak{X}$ into $\mathfrak{Y}$. When $K$ is given, let $\kappa_{K}$ be defined by

$$
\kappa_{K}(x \mid t)=K(t) x(t) .
$$

When $\kappa$ is given, let $K_{\kappa}$ be defined by

$$
K_{\kappa}(t) u=\kappa\left(x_{u} \mid t\right)
$$

where $x_{u}(t)=u$ for all $t$. Let

$$
M_{0}(K)=\sup \{\|K(t)\| \mid t \in \mathfrak{T}\} .
$$

As a consequence of the Uniform Boundedness Theorem of Hildebrandt (see also [6, p. 26]) we have the following condition for the finiteness of $M_{0}(K)\left({ }^{3}\right)$.

Lemma. If $K(t) u$ is a bounded function of $t$ for each fixed $u \in \mathfrak{U}$, then $M_{0}(K)$ $<\infty$.

We can now state the following relations between $K$ and $\kappa_{K}$, and between $\kappa$ and $K_{\kappa}$.

THEOREM 1. (a) If $K: \mathfrak{T} \rightarrow \Omega$ is continuous with the strong topology in $\Omega$, and $M_{0}(K)<\infty$, then $\kappa_{K}$ is a linear continuous transformation of $\mathfrak{X}$ into $\mathfrak{Y}$, and $\left\|\kappa_{K}\right\|=M_{0}(K)$. Moreover, if $\lambda=\kappa_{K}$, then $K_{\lambda}=K$.

(b) If $\kappa$ is a linear continuous transformation of $\mathfrak{X}$ into $\mathfrak{Y}$, then $K_{\mathfrak{k}}$ maps $\mathfrak{I}$ into $\Omega$, is continuous with the strong topology in $\Omega$, and $M_{0}\left(K_{\kappa}\right) \leqq\|\kappa\|$. Moreover if $L=K_{\kappa}$, then $\kappa_{L}=\kappa$ if and only if, for each $t \in \mathfrak{T}, x_{1}(t)=x_{2}(t)$ implies $\kappa\left(x_{1} \mid t\right)=\kappa\left(x_{2} \mid t\right)$. When this last condition holds, and $\kappa$ maps $\mathfrak{X}$ onto $\mathfrak{Y}$, then $K_{\kappa}$ maps $\mathfrak{T}$ into $\Omega_{0}$.

The verification of these statements is straightforward, and will be omitted. A simple example in which $\kappa$ maps $\mathfrak{X}$ onto $\mathfrak{Y}$, but $K_{\kappa}$ does not map $\mathfrak{I}$ into $\Omega_{0}$, is obtained by taking $\mathfrak{T}=[0,1], \mathfrak{U}=\mathfrak{B}=$ reals, $\kappa(x \mid t)=x(t)$ $-t \int_{0}^{1} x(s) d s$. Here $\kappa^{-1}(y \mid t)=y(t)+2 t \int_{0}^{1} y(s) d s, K_{\kappa}(1)=0$.

It is natural to ask about conditions for the mapping $K$ to be continuous with the uniform topology in $\Omega$. The following answer is readily verified.

(3) We note that in the lemma the completeness of the space $\mathfrak{B}$ is not needed, and in Theorem 1 neither $\mathfrak{U}$ nor $\mathfrak{B}$ needs to be complete. 
Theorem 2. Let $\mathfrak{X}_{1}$ denote the set of constant functions in $\mathfrak{X}$ with norm equal to 1 . Let $\kappa$ be a linear continuous transformation of $\mathfrak{X}$ into $\mathfrak{Y}$. Then the associated mapping $K_{\kappa}$ is continuous with the uniform topology in $\Omega$ if and only if the set of functions $\kappa\left(\mathfrak{X}_{1}\right)$ is equicontinuous on $\mathfrak{T}$.

Since the concepts of equicontinuity and compactness are sometimes related, the next theorem seems rather natural.

THEOREM 3. If $\kappa$ is a completely continuous linear transformation on $\mathfrak{X}$ to $\mathfrak{Y}$, then the associated mapping $K_{\kappa}$ is continuous with the uniform topology in $\Omega$, and each $K_{\kappa}(t)$ is a completely continuous linear transformation on $\mathfrak{U}$ to $\mathfrak{B}$.

Proof. In view of Theorem 2, to prove the first assertion in the theorem it is sufficient to prove that $\kappa\left(\mathfrak{X}_{1}\right)$ is an equicontinuous family of functions in $\mathfrak{Y}$ ). Since $\mathfrak{X}_{1}$ is a bounded family of functions in $\mathfrak{X}$, the hypotheses imply that $\kappa\left(\mathfrak{X}_{1}\right)$ is totally bounded in $\mathfrak{V}$. Hence, given any $\epsilon>0$, there exists a finite set of functions $\left\{z_{j}\right\}$ in $\mathfrak{V}$ so that for each $x_{u} \in \mathfrak{X}_{1}$ there is at least one $j$ for which $\left\|\kappa\left(x_{u}\right)-z_{j}\right\|_{y}<\epsilon / 3$. For fixed $t_{0}$ and for each $j$, there exists a neighborhood $U_{j}\left(t_{0}\right)$ so that $t \in U_{j}\left(t_{0}\right)$ implies that $\left\|z_{j}(t)-z_{j}\left(t_{0}\right)\right\|_{v}<\epsilon / 3$. Since there are only a finite number of $z_{j}$, we can take $U\left(t_{0}\right)=\bigcap_{j} U_{j}\left(t_{0}\right)$. Then $t \in U\left(t_{0}\right)$ implies that for any $x_{u} \in \mathfrak{X}_{1}$ we have

$$
\begin{aligned}
&\left\|\kappa\left(x_{u} \mid t\right)-\kappa\left(x_{u} \mid t_{0}\right)\right\| \\
& \leqq\left\|\kappa\left(x_{u} \mid t\right)-z_{j}(t)\right\|+\left\|z_{j}(t)-z_{j}\left(t_{0}\right)\right\|+\left\|z_{j}\left(t_{0}\right)-\kappa\left(x_{u} \mid t_{0}\right)\right\|<\epsilon
\end{aligned}
$$

for some one of the $z_{j}$. So $\kappa\left(\mathfrak{X}_{1}\right)$ is equicontinuous and $K_{k}$ is continuous with the uniform topology in $\Omega$. The final statement of the theorem is immediate.

The converse of Theorem 3 is not true. That is, if $K(t)$ is completely continuous for each $t$ and $K$ is continuous with the uniform topology in $\Omega$, then it does not follow that the induced mapping $\kappa_{K}$ is completely continuous. For example, if $\mathfrak{U}$ and $\mathfrak{B}$ are chosen to be the real line, $\mathfrak{T}$ is the closed unit interval, and $K(t)$ is the identity map for all $t$, then each $K(t)$ is completely continuous. However, $\kappa$ is the identity transformation on $C[0,1]$ and is not completely continuous.

Evidently it is easier to carry the properties from $\kappa$ to $K_{\kappa}$. In the next section we shall go the other way and prove a converse to the final state ment in Theorem 1 (b).

3. Induced transformations mapping $\mathfrak{X}$ onto $\mathfrak{Y}$. If $L \in \Omega_{0}$, that is, $L$ is a linear continuous transformation mapping $\mathfrak{U}$ onto $\mathfrak{B}$, then from a result of Banach $[1$, p. 38] it follows that

$$
I(L)=\sup _{\|\| \|=1}\left\{\inf _{L u=0 .}\|u\|\right\}
$$

is finite. In the special case where $L$ is one-to-one, $I(L)=\left\|\dot{L}^{-1}\right\|$. It is easily seen that $I(L)$ is upper semicontinuous on $\Omega_{0}$ in the uniform topology. [See 5 , 
Theorem 1.] For a mapping $K$ of the space $\mathfrak{T}$ into $\Omega_{0}$, we shall be concerned with the number $M_{0}$ defined by (2), and with the number

$$
N_{0}=\sup \{I(K(t)) \mid t \in \mathfrak{I}\} .
$$

When $K$ is continuous in the uniform topology of $\Omega$, and $\mathfrak{I}$ is sequentially compact, then $M_{0}$ and $N_{0}$ are automatically finite.

THEOREM 4. Suppose that the space $\mathfrak{I}$ is paracompact, that $K$ is a mapping of $\mathfrak{T}$ into $\Omega_{0}$ which is continuous in the uniform topology, and that $M_{0}$ and $N_{0}$ are finite. Then the induced transformation $\kappa$ defined by (1) maps $\mathfrak{X}$ onto all of $\mathfrak{Y}$. Moreover, if $N>N_{0}, y \in \mathfrak{Y}$, and $\epsilon>0$, then there exists an $x \in \mathfrak{X}$ for which $\kappa(x)=y$ and $\|x(t)\| \leqq N\|y(t)\|+\epsilon$ for all $t \in \mathfrak{T}$.

Proof. Suppose that the theorem is proved for all $y \in \mathfrak{Y}$ such that $\|y\|$ $\leqq 1$. Then for an arbitrary $y \in \mathfrak{Y}$ we take $y^{*}(t)=y(t)$ when $\|y(t)\| \leqq 1$ and $y^{*}(t)=y(t) /\|y(t)\|$ when $\|y(t)\| \geqq 1$. By our supposition there exists an $x^{*} \in \mathfrak{X}$ so that $\kappa\left(x^{*}\right)=y^{*}$ and $\left\|x^{*}(t)\right\| \leqq N\left\|y^{*}(t)\right\|+\epsilon$ for all $t \in \mathfrak{T}$. We now put $x(t)=x^{*}(t)$ when $\|y(t)\| \leqq 1$ and $x(t)=\|y(t)\| x^{*}(t)$ when $\|y(t)\| \geqq 1$. It is easily verified that $x(t)$ satisfies the conclusions of the theorem with $N$ replaced by $N+\epsilon$. Since $N$ and $\epsilon$ are subject only to the restrictions $N>N_{0}$ and $\epsilon>0$, we see that it suffices to prove the theorem under the hypothesis that $\|y\| \leqq 1$.

Suppose, then, that we are given such an element $y \in \mathfrak{Y}$ as well as the two numbers $N$ and $\epsilon$. Take $q>2(N+\epsilon+1)^{2}$. For each $t_{0} \in \mathfrak{T}$ and for any positive integer $r$, the continuity of $y$ and $K$ as functions of $t$ implies that there exists an open neighborhood $V_{r}\left(t_{0}\right)$ of $t_{0}$ such that if $t^{\prime} \in V_{r}\left(t_{0}\right)$, then

$$
\begin{gathered}
\left\|y\left(t^{\prime}\right)-y\left(t_{0}\right)\right\|<\epsilon / 2 q^{r}, \\
\left\|K\left(t^{\prime}\right)-K\left(t_{0}\right)\right\|<\epsilon / 2 q^{r} .
\end{gathered}
$$

We cover $\mathfrak{T}$ by the open sets $\left\{V_{1}(t) \mid t \in \mathfrak{T}\right\}$. By the paracompactness of $\mathfrak{T}$ there exists an open covering $\mathfrak{P}_{1}=\left\{P_{1, v}\right\}$ such that each $P_{1, \nu} \subset V_{1}\left(t_{1, v}\right)$ for some point $t_{1, v}$, and so that the covering $\mathfrak{P}_{1}$ is neighborhood-finite. Let $\left\{\phi_{1, v}\right\}$ be a continuous partition of unity subordinate to $\mathfrak{B}_{1}$, so that $\phi_{1, \nu}$ vanishes outside of $V_{1}\left(t_{1, v}\right)$ (since by construction it vanishes outside of $\left.P_{1, v}\right)$.

Define $y_{1}(t)=\sum, \phi_{1, v}(t) y\left(t_{1, v}\right)$. By construction of the $\phi$ 's we have that $\phi_{1, v}$ vanishes unless $t$ is such that $\left\|y\left(t_{1, v}\right)-y(t)\right\|<\epsilon / 2 q$. Hence we have that $\left\|y_{1}(t)-y(t)\right\|=\left\|\sum \phi_{1, v}(t)\left\{y\left(t_{1, v}\right)-y(t)\right\}\right\| \leqq \sum \phi_{1, v}(t)\left\|y\left(t_{1, v}\right)-y(t)\right\|<\epsilon / 2 q$ for all $t \in \mathfrak{T}$. So we have for all $t$ :

$$
\left\|y_{1}(t)-y(t)\right\|<\epsilon / 2 q .
$$

Since $K(t)$ maps onto $\mathfrak{B}$ for each $t$, then for each $\nu$ there exists a $u_{1, \nu}$ so that $K^{-}\left(t_{1, v}\right) u_{1, v}=y\left(t_{1, v}\right)$ and

$$
\left\|u_{1 . \nu}\right\|<x\left\|y\left(t_{1, i}\right)\right\|
$$


We define $x_{1}(t)=\sum \phi_{1, \nu}(t) u_{1, \nu}$. Then

$$
\left\|x_{1}(t)\right\|=\left\|\sum \phi_{1, \nu}(t) u_{1, v}\right\| \leqq \sum \phi_{1, v}(t)\left\|u_{1, v}\right\|<N \sum \phi_{1, v}(t)\left\|y\left(t_{1, v}\right)\right\| .
$$

But from $\left(3_{1}\right)$ and the triangle inequality, we have for $t \in V_{1}\left(t_{1, v}\right)$ that

$$
\left|\left\|y\left(t_{1, v}\right)\right\|-\|y(t)\|\right| \leqq\left\|y\left(t_{1, v}\right)-y(t)\right\|<\epsilon / 2 q .
$$

So we get that $\left\|y\left(t_{1, v}\right)\right\|<\|y(t)\|+\epsilon / 2 q$. Inserting this in the preceding and using the definition of $q$ to show that $N / q<1$, we have for all $t$ :

$$
\left\|x_{1}(t)\right\|<N\|y(t)\|+\epsilon / 2 \text {. }
$$

Also $\left\|y_{1}(t)-K(t) x_{1}(t)\right\|=\left\|\sum \phi_{1, v}(t) y\left(t_{1, v}\right)-K(t)\left[\sum \phi_{1, v}(t) u_{1, v}\right]\right\|=\| \sum \phi_{1, v}(t)$ . $\left\{K\left(t_{1, \nu}\right) u_{1, \nu}-K(t) u_{1, \nu}\right\}\left\|\leqq \sum \phi_{1, \nu}(t)\right\| K\left(t_{1, \nu}\right) u_{1, \nu}-K(t) u_{1, \nu} \|$. Since $\phi_{1, \nu}(t)=0$ except possibly for those $t$ for which $\left(4_{1}\right)$ holds, we see with the help of $\left(6_{1}\right)$ and $\left(5_{1}\right)$ that the last expression is less than

$$
\begin{aligned}
\sum \phi_{1, \nu}(t)\left\|u_{1, \nu}\right\| \epsilon / 2 q & <(N \epsilon / 2 q) \sum \phi_{1, \nu}(t)\left\|y\left(t_{1, \nu}\right)\right\| \\
& <(N \epsilon / 2 q) \sum \phi_{1, \nu}(t)\{\|y(t)\|+\epsilon / 2 q\} \\
& \leqq(N \epsilon / 2 q)(1+\epsilon / 2 q)=(\epsilon / 2 q)(N+N \epsilon / 2 q) .
\end{aligned}
$$

Combining this with $\left(5_{1}\right)$ and the definition of $q$, we have

$$
\begin{aligned}
\left\|y(t)-K(t) x_{1}(t)\right\| & <(\epsilon / 2 q)(N+N \epsilon / 2 q+1)<(\epsilon / 2 q)(N+\epsilon+1) \\
& <\epsilon / 4(N+\epsilon+1)<\epsilon / 4 N .
\end{aligned}
$$

Hence we have shown that, for all $t$ :

$$
\left\|y(t)-K(t) x_{1}(t)\right\|<\epsilon / 2^{2} N .
$$

Suppose that for each positive integer $r \leqq m$ we have chosen a pair of bounded continuous functions $y_{r}$ and $x_{r}$ so that for each $t \in \mathfrak{T}$ the following inequalities are satisfied:

$$
\begin{gathered}
\left\|y_{r}(t)-y(t)\right\|<\epsilon / 2 q^{r}, \\
\left\|x_{r}(t)\right\|<N\|y(t)\|+\epsilon / 2+\cdots+\epsilon / 2^{r}, \\
\left\|y(t)-K(t) x_{r}(t)\right\|<\epsilon / 2^{r+1} N \\
\left\|x_{r}(t)-x_{r-1}(t)\right\|<\epsilon / 2^{r}
\end{gathered}
$$

We shall show that we can construct $y_{m+1}$ and $x_{m+1}$ so that these same inequalities are true with $r$ replaced by $m+1$.

For any $t_{0}$, since $K\left(t_{0}\right)$ maps onto $\mathfrak{B}$, we can choose a point $u_{0} \in \mathfrak{U}$ so that $K\left(t_{0}\right) u_{0}=y\left(t_{0}\right)$ and

$$
\begin{aligned}
\left\|u_{0}-x_{m}\left(t_{0}\right)\right\| & \leqq N\left\|K\left(t_{0}\right)\left[u_{0}-x_{m}\left(t_{0}\right)\right]\right\| \\
& =N\left\|y\left(t_{0}\right)-K\left(t_{0}\right) x_{m}\left(t_{0}\right)\right\|<\epsilon / 2^{m+1},
\end{aligned}
$$


the last inequality being true by virtue of $\left(8_{m}\right)$. Consider the set of points $\left\{t \mid\left\|u_{0}-x_{m}(t)\right\|<\epsilon / 2^{m+1}\right\}$. From the preceding remark this set is nonvoid; since $x_{m}$ is continuous, it is open. Hence there exists an open neighborhood $W_{m+1}\left(t_{0}\right)$ of $t_{0}$ which is contained in the set $V_{m+1}\left(t_{0}\right) \cap\left\{t \mid\left\|u_{0}-x_{m}(t)\right\|<\epsilon / 2^{m+1}\right\}$.

For each point $t \in \mathfrak{T}$ we choose a point $u$ and an open set $W_{m+1}(t)$ as prescribed in the preceding paragraph. The family of open sets $\left\{W_{m+1}(t) \mid t \in \mathfrak{T}\right\}$ form an open covering of $\mathfrak{T}$; by paracompactness there is an open covering $\mathfrak{P}_{m+1}=\left\{P_{m+1, \nu}\right\}$ which is neighborhood-finite and each of whose sets is contained in $W_{m+1}(t)$ for some $t$ (which we shall henceforth label as $t_{m+1, \nu}$ ). As before we construct a continuous partition of unity $\left\{\phi_{m+1, \nu}\right\}$ subordinate to the covering $\mathfrak{B}_{m+1}$. We then define

$$
y_{m+1}(t)=\sum \phi_{m+1, \nu}(t) y\left(t_{m+1, \nu}\right), \quad x_{m+1}(t)=\sum \phi_{m+1, \nu}(t) u_{m+1, \nu},
$$

where the point $u_{m+1, \nu}$ is the point chosen to correspond to the point $t_{m+1, \nu}$ by the process outlined above. Since $\phi_{m+1, \nu}$ vanishes outside $P_{m+1, \nu}$, we see from $\left(3_{m+1}\right)$ that

$$
\left(5_{m+1}\right) \quad\left\|y_{m+1}(t)-y(t)\right\|<\epsilon / 2 q^{m+1}
$$

for all $t$. Since $\phi_{m+1,}$ vanishes except possibly for those $t$ for which $\left(6_{m+1}\right)$ holds, we have

$$
\begin{aligned}
\left\|x_{m+1}(t)-x_{m}(t)\right\| & =\left\|\sum \phi_{m+1, \nu}(t) u_{m+1, \nu}-x_{m}(t)\right\| \\
& =\left\|\sum \phi_{m+1, \nu}(t)\left\{u_{m+1, \nu}-x_{m}(t)\right\}\right\| \\
& \leqq \sum \phi_{m+1, \nu}(t)\left\|u_{m+1, \nu}-x_{m}(t)\right\|<\epsilon / 2^{m+1} .
\end{aligned}
$$

Hence for all $t$, we have

$\left(9_{m+1}\right)$

$$
\left\|x_{m+1}(t)-x_{m}(t)\right\|<\epsilon / 2^{m+1} .
$$

Combining this with $\left(7_{m}\right)$ we get

$\left(7_{m+1}\right)$

$$
\left\|x_{m+1}(t)\right\|<N\|y(t)\|+\epsilon / 2+\cdots+\epsilon / 2^{m}+\epsilon / 2^{m+1}
$$

for all $t \in \mathfrak{T}$. Also

$$
\begin{array}{r}
\left\|y_{m+1}(t)-K(t) x_{m+1}(t)\right\|=\left\|\sum \phi_{m+1, \nu}(t)\left\{y\left(t_{m+1, \nu}\right)-K(t) u_{m+1, \nu}\right\}\right\| \\
=\left\|\sum \phi_{m+1, \nu}(t)\left\{K\left(t_{m+1, \nu}\right) u_{m+1, \nu}-K(t) u_{m+1, \nu}\right\}\right\| .
\end{array}
$$

Now by $\left(6_{m+1}\right)$ and $\left(7_{m}\right),\left\|u_{m+1, \nu}\right\|<N\left\|y\left(t_{m+1, \nu}\right)\right\|+\epsilon \leqq N+\epsilon$, and since $\phi_{m+1, \nu}$ vanishes outside of the set where $\left(4_{m+1}\right)$ holds, $\left\|y_{m+1}(t)-K(t) x_{m+1}(t)\right\|$ $<(N+\epsilon) \epsilon / 2 q^{m+1}$. By combining this inequality with $\left(5_{m+1}\right)$ we obtain

$$
\left\|y(t)-K(t) x_{m+1}(t)\right\|<\epsilon / 2^{m+2} N,
$$

since $\epsilon(N+\epsilon+1) / 2 q^{m+1}<\epsilon / 2^{m+2} N$.

Thus we obtain by the inductive process described above two infinite sequences $\left\{y_{r}\right\}$ and $\left\{x_{r}\right\}$. From (9), it follows that $\left\{x_{r}\right\}$ is a Cauchy se- 
quence in $\mathfrak{X}$, and so there exists a function $x \in \mathfrak{X}$ which is the limit of this sequence. In terms of the induced transformation $\kappa,\left(8_{r}\right)$ states that $\left\|y-\kappa\left(x_{r}\right)\right\|<\epsilon / 2^{r+1} N$. Hence $\kappa\left(x_{r}\right) \rightarrow y$ in the space $\mathfrak{Y}$. But since $\kappa$ is a continuous function on $\mathfrak{X}$, we have $\kappa\left(x_{r}\right) \rightarrow \kappa(x)$ in the space $\mathfrak{Y}$. Consequently $y=\kappa(x)$; that is, $\kappa$ maps $\mathfrak{X}$ onto $\mathfrak{Y}$.

Passing to the limit in $\left(7_{r}\right)$ we see that $\|x(t)\|<N\|y(t)\|+\epsilon$ for all $t \in \mathfrak{T}$. This completes the proof of the theorem.

The next corollary allows one to drop the $\epsilon$ in the estimate on the magnitude of $x(t)$ given in the final inequality of the theorem.

COROLLARY. With the hypotheses of the theorem, given any $N>N_{0}$ and any $y \in \mathfrak{Y}$ for which the open subset of $\mathfrak{I}$ where $y(t) \neq 0$ is paracompact, then the final inequality of the theorem may be strengthened to read $\|x(t)\| \leqq N\|y(t)\|$ for all $t \in \mathfrak{T}$. In particular this is true for arbitrary $y \in \mathfrak{Y}$ when $\mathfrak{I}$ is a metric space.

Proof. If $y(t) \neq 0$ on $\mathfrak{I}$, we can define the function $y^{*}(t)=y(t) /\|y(t)\|$ for all values of $t$, and $\left\|y^{*}(t)\right\|=1$. Pick $\epsilon>0$ so small that $N_{0}<N-\epsilon$, and let $N^{*}=N-\epsilon$. Applying the theorem to $N^{*}, y^{*}$, and $\epsilon$ we get an $x^{*} \in X$ such that $\kappa\left(x^{*}\right)=y^{*}$ and $\left\|x^{*}(t)\right\|<N^{*}\left\|y^{*}(t)\right\|+\epsilon=N-\epsilon+\epsilon=N$. From this, by defining $x(t)=\|y(t)\| x^{*}(t)$, we get $\kappa(x)=y$ and $\|x(t)\| \leqq N\|y(t)\|$.

If the set $\mathfrak{I}_{0}=\{t \mid y(t) \neq 0\}$ is paracompact, we can consider the function $y$ restricted to $\mathfrak{I}_{0}$, in which case the preceding remarks are valid and we get an $x$ obeying the stronger inequality on $\mathfrak{I}_{0}$. Extending the domain of definition of this $x$ to all of $\mathfrak{I}$ by setting it equal to zero outside of $\mathfrak{I}_{0}$ gives a continuous function on $\mathfrak{I}$ which satisfies the two conditions.

In passing, we note that the preceding result generalizes Theorem 4 of $[1$, p. 40$]$, in two directions.

4. Nonlinear transformations. The next theorem is a corollary of Theorem 1 of [5], except for the pointwise inequality on the solution. The proof is along the same lines, and is omitted. We recall that $\mathfrak{X}_{B}$ and $\mathfrak{Y}_{B}$ denote the Banach spaces composed of the bounded functions in $\mathfrak{X}$ and $\mathfrak{Z}$ respectively.

THEOREM 5. Let $\lambda$ be a linear continuous mapping of $\mathfrak{X}_{B}$ onto $\mathfrak{Y}_{B}$, for which there exists a constant $N$ with the property that for each $y \in \mathfrak{Y}_{B}$ the equation $y=\lambda(x)$ has a solution $x \in \mathfrak{X}_{B}$ with $\|x(t)\| \leqq N\|y(t)\|$ on $\mathfrak{T}$. Let $\Gamma(x)$ be defined and in $\mathfrak{Y}_{B}$ for $\|x\|<\delta$, and satisfy $\Gamma(0)=0$, and

$$
\left\|\Gamma\left(x_{1} \mid t\right)-\Gamma\left(x_{2} \mid t\right)-\lambda\left(x_{1}-x_{2} \mid t\right)\right\| \leqq Q\left\|x_{1}(t)-x_{2}(t)\right\|
$$

for $t \in \mathfrak{T},\left\|x_{i}\right\|<\delta$, where $N Q<1$.

Then for each $y \in \mathfrak{Y}_{B}$ with $\|y\|<\delta(1-N Q) / N$ the equation $y=\Gamma(x)$ has a solution $x \in \mathfrak{X}_{B}$ with

$$
\|x(t)\| \leqq\|y(t)\| N /(1-N Q) .
$$

In case $\delta=\infty$, Theorem 5 yields the result that $\Gamma$ maps $\mathfrak{X}_{B}$ onto all of 
$\mathfrak{V}_{B}$. If also $\Gamma$ is positively homogeneous of degree one, and the value $\Gamma(x \mid t)$ of the function $\Gamma(x)$ at the place $t$ depends only on $x(t)$, then $\Gamma$ maps $\mathfrak{X}$ onto $\mathfrak{y}$. This may be shown by the device used in the proof of Theorem 4 , namely, by replacing $y(t)$ by $y(t) /\|y(t)\|$ at values of $t$ where $\|y(t)\|>1$.

-In Theorem 4 the mapping $K$ was required to be continuous in the uniform topology of $\Omega$. The next corollary extends part of the results of Theorem 4 to some mappings which are only continuous in the strong topology of $\Omega$.

Corollary. Let $K$ be a mapping satisfying the conditions of Theorem 4, and let $\kappa$ be the induced linear transformation of $\mathfrak{X}$ onto $\mathfrak{Y}$. Let $A$ be another mapping of $\mathfrak{T}$ into $\Omega$, continuous in the strong topology of $\Omega$, and let $\alpha$ be the corresponding induced linear transformation of $\mathfrak{X}$ into $\mathfrak{Y}$. Suppose in addition that

$$
\|A(t)\| \leqq M_{0}<1 / N_{0}, \quad N_{0}=\sup I(K(t)),
$$

and that $\mathfrak{I}$ is a metric space. Then $\kappa-\alpha$ maps $\mathfrak{X}$ onto all of $\mathfrak{Y}$, and if $0<N_{0} M_{0}$ $<N M_{0}<1, \kappa x-\alpha x=y$ has a solution $x$ with

$$
\|x(t)\| \leqq\|y(t)\| N /\left(1-N M_{0}\right) .
$$

From Theorem 5 we can deduce the existence of a continuous solution $u=x(t)$ of an equation $G(u, t)=0$ even when the solution is not uniquely determined. This result is embodied in Theorem 6 , and a special case in the following corollary.

THEOREM 6. Let $L$ be a linear continuous transformation of $\mathfrak{u}$ onto the whole of $\mathfrak{B}$, and let $0<N Q<1$, where $N>I(L)$. Let $\mathfrak{T}$ be a metric space, and let $G(u, t)$ be continuous for $\|u\|<\delta, t \in \mathfrak{T}$, with values in $\mathfrak{B}$. Suppose also that

$$
\begin{gathered}
\left\|G\left(u_{1}, t\right)-G\left(u_{2}, t\right)-L\left(u_{1}-u_{2}\right)\right\| \leqq Q\left\|u_{1}-u_{2}\right\| \quad \text { for }\left\|u_{i}\right\|<\delta, t \in \mathfrak{T}, \\
\|G(0, t)\|<(1-N Q) \delta / N \text { on } \mathfrak{T}, \\
G\left(0, t_{0}\right)=0 .
\end{gathered}
$$

Then there is a continuous function $x(t)$ defined on $\mathfrak{I}$ such that

$$
G(x(t), t)=0, \quad x\left(t_{0}\right)=0, \quad\|x(t)\| \leqq\|G(0, t)\| N /(1-N Q) .
$$

Proof. Let $\lambda(x \mid t)=L(x(t)), \Gamma(x \mid t)=G(x(t), t)-G(0, t), y(t)=-G(0, t)$. Then it follows from Theorem 4 that Theorem 5 is applicable.

Corollary. Let $\mathfrak{T}$ be a metric space, and suppose $G(u, t)$ and its partial differential $d_{u} G(u, t ; d u)$ are continuous for $\|u\|<\delta, t \in \mathfrak{T}$, uniformly for $\|d u\|$ $=1$. Suppose also that $G\left(0, t_{0}\right)=0$, and that $d_{u} G\left(0, t_{0} ; d u\right)$ maps $\mathfrak{U}$ onto the whole space $\mathfrak{B}$. Then there exists a number $\epsilon>0$ and a continuous function $x(t)$ defined for dist $\left(t, t_{0}\right)<\epsilon$ such that $G(x(t), t)=0, x\left(t_{0}\right)=0$.

5. Equations of Volterra type. The next theorem includes results on the 
existence of solutions for integral equations and for ordinary differential equations in cases when the solution is not unique.

THEOREM 7. Suppose that $\mathfrak{T}$ is a linear interval $[0, T]$, and let $\lambda$ satisfy the conditions of Theorem 5. Let $\alpha(x)$ be defined and in $\mathfrak{Y}$ for $\|x\|<\delta$, and satisfy $\alpha(0)=0$, and

$$
\left\|\alpha\left(x_{1} \mid t\right)-\alpha\left(x_{2} \mid t\right)\right\| \leqq Q \int_{0}^{t}\left\|x_{1}(s)-x_{2}(s)\right\| d s
$$

for $t \in \mathfrak{T},\left\|x_{i}\right\|<\delta$, where $Q>0$.

Then for each $y \in \mathfrak{V}$ with $\|y\|<\delta e^{-N Q T} / N$ the equation

$$
\lambda(x)-\alpha(x)=y
$$

has a solution $x \in \mathfrak{X}$ satisfying the two inequalities

$$
\begin{aligned}
& \|x(t)\| \leqq N\|y(t)\|+N\|y\|\left\{e^{N Q t}-1\right\} \leqq N\|y\| e^{N Q T}, \\
& \|x(t)\| \leqq N\|y(t)\|+N^{2} Q e^{N Q t} \int_{0}^{t}\|y(s)\| d s .
\end{aligned}
$$

Proof. Since $\mathfrak{I}$ is compact, we have $\mathfrak{X}_{B}=\mathfrak{X}, \mathfrak{Y}_{B}=\mathfrak{Y}$. From the assumptions on $\lambda$ and $\alpha$ it is clear that there exist solutions $x_{0}, x_{1}, x_{2}, \ldots$ of the equations

$$
\lambda x_{0}=y, \quad \lambda x_{n}=y+\alpha x_{n-1},
$$

satisfying the inequalities

$$
\begin{aligned}
\left\|x_{0}(t)\right\| & \leqq N\|y(t)\| \\
\left\|x_{n}(t)-x_{n-1}(t)\right\| & \leqq N\|y\|(N Q t)^{n} / n ! \\
\left\|x_{n}(t)-x_{n-1}(t)\right\| & \leqq N^{2} Q \int_{0}^{t}\|y(s)\| d s(N Q t)^{n-1} /(n-1) !,
\end{aligned}
$$

for $0 \leqq t \leqq T$. Thus the approximations $x_{n}$ lie in the domain of $\alpha$, and converge uniformly to a solution satisfying the stated inequalities.

We note that the inequality (12) of the theorem implies that

$$
\int_{0}^{t}\|x(s)\| d s \leqq\left(N+N e^{N Q t}\right) \int_{0}^{t}\|y(s)\| d s,
$$

for all $t$.

6. Solutions of class $C^{\prime}$. In this section we shall suppose that $\mathfrak{I}$ is a linear interval $[0, T]$, and shall let $\mathfrak{X}^{\prime}$ and $\mathfrak{Y}^{\prime}$ denote the subspaces of $\mathfrak{X}$ and $\mathfrak{Y}$ composed of those functions which are of class $C^{\prime}$ on $\mathfrak{T}$. With the new norms

$$
\|x\|^{\prime}=\sup \left\|x^{\prime}(t)\right\|+\|x(0)\|, \quad\|y\|^{\prime}=\sup \left\|y^{\prime}(t)\right\|+\|y(0)\| .
$$

these are Banach spaces. 
TheOREм 8. Let $K(t)$ be of class $C^{\prime}$ on $\mathfrak{T}$ with values in $\Omega_{0}$. Then the induced transformation $\kappa$ maps $\mathfrak{X}^{\prime}$ onto all of $\mathfrak{Y}^{\prime}$ and the equation $\kappa x=y$ has a solution $x$ with

$$
\|x\|^{\prime} \leqq N(1+N Q) e^{N Q T}\|y\|^{\prime},
$$

where $N>N_{0}=\sup I(K(t)), Q=\sup \left\|K^{\prime}(t)\right\|$.

Proof. The equation

$$
K(t) x(t)=y(t)
$$

is equivalent to

$$
\begin{gathered}
K(0) x(0)=y(0) \\
K(t) x^{\prime}(t)+K^{\prime}(t)\left[x(0)+\int_{0}^{t} x^{\prime}(s) d s\right]=y^{\prime}(t) .
\end{gathered}
$$

(See [4, Theorem 4].) By hypothesis, equation (14) has a solution $x(0)$ with

$$
\|x(0)\| \leqq N\|y(0)\| \text {. }
$$

To equation (15) we apply Theorem 7 , with $x(t)$ replaced by $x^{\prime}(t)$,

$$
\lambda\left(x^{\prime} \mid t\right)=K(t) x^{\prime}(t), \quad \alpha\left(x^{\prime} \mid t\right)=-K^{\prime}(t) \int_{0}^{t} x^{\prime}(s) d s,
$$

and with $y(t)$ replaced by $y^{\prime}(t)-K^{\prime}(t) x(0)$. From the inequality (11) of Theorem 7, we find that a solution exists satisfying

$\left\|x^{\prime}(t)\right\| \leqq N e^{N Q T} \sup \left\|y^{\prime}(t)-K^{\prime}(t) x(0)\right\| \leqq N e^{N Q T}\left\{\sup \left\|y^{\prime}(t)\right\|+N Q\|y(0)\|\right\}$, and by combining this with (16) and the definition of $\|x\|^{\prime}$ and $\|y\|^{\prime}$, we obtain (13).

THEOREM 9. Let $G(u, t)$ be of class $C^{\prime \prime}$ for $\left\|u-u_{0}\right\|<\delta, t \in \mathfrak{T}$, and suppose that $d_{u} G\left(u_{0}, t ; d u\right)$ maps $\mathfrak{U}$ onto $\mathfrak{B}$ for each $t \in \mathfrak{T}$. Let $y_{0}(t)=G\left(u_{0}, t\right)$. Then for each $y \in \mathfrak{Y}^{\prime}$ with $\left\|y-y_{0}\right\|^{\prime}$ sufficiently small, there exists $x \in \mathfrak{X}^{\prime}$ such that $\left\|x(t)-u_{0}\right\|$ $<\delta$ and

$$
G(x(t), t)=y(t) .
$$

Proof. Let $\Gamma(x)$ be defined by

$$
\Gamma(x \mid t)=G(x(t), t)
$$

for $x \in \mathfrak{X}^{\prime}$ with $\left\|x(t)-u_{0}\right\|<\delta$. If we denote this domain of the function $\Gamma$ by $\mathfrak{X}_{0}^{\prime}$, it is straightforward to verify that $\Gamma$ is of class $C^{\prime}$ on $\mathfrak{X}_{0}^{\prime}$, with values in $\mathfrak{Y}^{\prime}$, and that its differential $d \Gamma(x ; d x)$ is given by

$$
d \Gamma(x ; d x \mid t)=d_{u} G(x(t), t ; d x(t)) \text {. }
$$


If we set $x_{0}(t)=u_{0}$, we see by Theorem 8 that $d \Gamma\left(x_{0} ; d x\right)$ maps $\mathfrak{X}^{\prime}$ onto $\mathfrak{Y}^{\prime}$. Then by Theorem 2 of [4], the desired result follows. It is easily seen that we may require that $\left\|x-x_{0}\right\|^{\prime}$ be arbitrarily small by taking $\left\|y-y_{0}\right\|^{\prime}$ sufficiently small.

7. Generalization of equations of Volterra type. Theorem 7 is here generalized in two ways-by removing most of the restrictions on the space $\mathfrak{T}$, and by modifying the Lipschitz condition (10) on the function $\alpha(x)$. We suppose that $\mathfrak{T}$ is the Cartesian product of two Hausdorff spaces $\Re$ and $\mathfrak{S}$. Corresponding to a real-valued nonnegative function $p(r)$ defined on $\Re$, and a function $x \in \mathfrak{X}_{B}$, we set

$$
\begin{array}{rlrl}
P & =\sup p(r), & & \\
\pi(x, \theta, s) & =\sup \|x(r, s)\| & & (\text { for } p(r) \leqq \theta) \\
& =0 & (\text { if } p(r)>\theta \text { for all } r) .
\end{array}
$$

We note that for each $x \in \mathfrak{X}_{B}, \pi$ is a finite nondecreasing function of $\theta$, and so is integrable on $[0, P]$ if $P$ is finite.

THEOREM 10. Let $\lambda$ be a linear transformation satisfying the conditions of Theorem 5. Let $\alpha(x)$ be defined and in $\mathfrak{Y}_{B}$ for $\|x\|<\delta$, and satisfy $\alpha(0)=0$ and

$$
\left\|\alpha\left(x_{1} \mid r, s\right)-\alpha\left(x_{2} \mid r, s\right)\right\| \leqq Q \int_{0}^{p(r)} \pi\left(x_{1}-x_{2}, \theta, s\right) d \theta
$$

for $\left\|x_{i}\right\|<\delta$, where $Q>0$. Suppose $P=\sup p(r)$ is finite.

Then for each $y \in \mathfrak{Y}_{B}$ with $\|y\|<\delta e^{-N P Q} / N$, the equation

$$
\lambda(x)-\alpha(x)=y
$$

has a solution $x \in \mathfrak{X}_{B}$ such that

$$
\|x(r, s)\| \leqq N e^{N P Q} \sup _{r}\|y(r, s)\| .
$$

The proof is like that for Theorem 7, and is omitted.

The following simple examples may serve to indicate possible applications of the last theorem. Let $\Re=\Re_{1} \times \Re_{2}$, where $\Re_{1}=\Re_{2}=[0,1]$, and let $A\left(r_{1}, r_{2}, \rho_{1}, \rho_{2}, s\right)$ be a continuous function on $\Re \times \Re \times \mathfrak{S}$.

1. $p\left(r_{1}, r_{2}\right)=r_{1}$

$$
\alpha\left(x \mid r_{1}, r_{2}, s\right)=\int_{0}^{r_{1}} \int_{0}^{1} A\left(r_{1}, r_{2}, \rho_{1}, \rho_{2}, s\right) x\left(\rho_{1}, \rho_{2}, s\right) d \rho_{2} d \rho_{1} .
$$

2. $p\left(r_{1}, r_{2}\right)=\sup \left\{r_{1}, r_{2}\right\}$,

$$
\alpha\left(x \mid r_{1}, r_{2}, s\right)=\int_{0}^{r_{1}} \int_{0}^{r_{2}} A\left(r_{1}, r_{2}, \rho_{1}, \rho_{2}, s\right) x\left(\rho_{1}, \rho_{2}, s\right) d \rho_{2} d \rho_{1} .
$$


We note that if $\alpha$ is itself a linear operator, then Theorem 10 states that the operator $\lambda-\alpha$ has properties like those of $\lambda$, in the following sense. We suppose that the spaces $\Re$ and $\subseteq$ are compact, so that $\mathfrak{X}_{B}=\mathfrak{X}$, etc. Then if $\mathfrak{X}_{R}$ denotes the space of continuous functions on $\mathfrak{R}$ to $\mathfrak{U}$, $\mathfrak{X}$ may be regarded as the space of continuous functions on $\mathfrak{S}$ to $\mathfrak{X}_{R}$, and

$$
\lambda(x)-\alpha(x)=y
$$

has a solution $x$ which, as a function of $s$, satisfies a pointwise bound. Thus if $\beta$ is another operator with $\beta(0)=0, p^{\prime}(s)$ is a nonnegative function defined on $\subseteq$,

$$
\pi^{\prime}\left(x, \theta^{\prime}\right)=\sup \|x(r, s)\| \quad \text { for } p^{\prime}(s) \leqq \theta^{\prime}, r \in \Re,
$$

and

$$
\left\|\beta\left(x_{1} \mid s\right)-\beta\left(x_{2} \mid s\right)\right\| \leqq Q^{\prime} \int_{0}^{p^{\prime}(s)} \pi^{\prime}\left(x_{1}-x_{2}, \theta^{\prime}\right) d \theta^{\prime},
$$

then Theorem 10 may be applied again to show the existence of a solution of $\lambda(x)-\alpha(x)-\beta(x)=y$.

A very special application of the last remark may be made to underdetermined systems of linear hyperbolic differential equations in two independent variables in which only the mixed second derivatives appear. To be explicit, consider the system

$$
l_{i j} \frac{\partial^{2} z_{j}}{\partial r \partial s}-a_{i j} \frac{\partial z_{j}}{\partial s}-b_{i j} \frac{\partial z_{j}}{\partial r}-c_{i j} z_{j}=d_{i},
$$

with prescribed values for $z_{j}(r, 0), z_{j}(0, s)$, where $i=1, \cdots, m ; j=1, \cdots$, $n>m$, and the terms are summed on the repeated index $j$. The coefficients $l_{i j}, a_{i j}, b_{i j}, c_{i j}, d_{i}$ are understood to be continuous functions of $(r, s)$ on a rectangular interval containing the origin. If we set

$$
\begin{aligned}
x_{j} & =\frac{\partial^{2} z_{j}}{\partial r \partial s}, \\
\lambda_{i}(x \mid r, s) & =l_{i j}(r, s) x_{j}(r, s), \\
\alpha_{i}(x \mid r, s) & =a_{i j}(r, s) \int_{0}^{r} x_{j}(\rho, s) d \rho \\
\beta_{i}(x \mid r, s) & =b_{i j}(r, s) \int_{0}^{s} x_{j}(r, \sigma) d \sigma+c_{i j}(r, s) \int_{0}^{r} \int_{0}^{s} x_{j}(\rho, \sigma) d \sigma d \rho,
\end{aligned}
$$

and group the remaining terms to form a function $y_{j}(r, s)$, we see that the conditions of the preceding discussion are satisfied, provided the matrix $\left(l_{i j}\right)$ has rank $m$ at every point. With the help of Theorem 2 of [4], an embed. ding theorem for nonlinear equations may then be secured. 


\section{REFERENCES}

1. S. Banach, Théorie des opérations linéaires, Warsaw, 1932.

2. N. Bourbaki, Topologie générale, chap. 9, Paris, 1948.

3. J. A. Dieudonne, Une généralisation des espaces compacts, J. Math. Pures Appl. vol. 23 (1944) pp. 65-76.

4. L. M. Graves, Riemann integration and Taylor's theorem in general analysis, Trans. Amer. Math. Soc. vol. 29 (1927) pp. 163-177.

5. —— Some mapping theorems, Duke Math. J. vol. 17 (1950) pp. 111-114.

6. E. Hille, Functional analysis and semi-groups, Amer. Math. Soc. Colloquium Publications, vol. 31, 1948.

7. A. H. Stone, Paracompactness and product spaces, Bull. Amer. Math. Soc. vol. 54 (1948) pp. 977-982.

8. S. Lefschetz, Algebraic topology, Amer. Math. Soc. Colloquium Publications, vol. 27, 1942.

University of Chicago

Chicago, Ill. 